\section{Genetic polymorphism of hOGG1 and risk of pterygium in Chinese}

\author{
Abstract \\ Purpose Ultraviolet irradiation is known to \\ cause oxidative DNA damage and is thought \\ to be a major factor implicated in the \\ pathogenesis of pterygium. The highly \\ mutagenic 8-hydroxy-2'-deoxyguanosine, a \\ marker for the evaluation of photo-oxidative \\ DNA damage, can be repaired by human \\ 8-oxoguanine glycosylase I (hOGG1). A \\ transition of $\mathrm{C}$ to $\mathrm{G}$ at nucleotide position 1245 \\ in exon 7 of the hOGG1 gene is associated \\ with the substitution of cysteine for serine at \\ codon 326. In this study, we investigated the \\ association of the hOGG1 Ser326Cys \\ polymorphism with pterygium in a Chinese \\ population. \\ Methods In all, 70 patients and 86 controls \\ were enrolled in this study. The Ser326Cys \\ polymorphism was determined by the \\ polymerase chain reaction-restriction \\ fragment-length polymorphism analysis. The \\ association between this genetic \\ polymorphism and risk of pterygium was \\ examined by $\chi^{2}$-test and logistic regression. \\ Results The allelic frequencies for the Ser \\ and Cys variants of hOGG1 gene were not \\ significantly different between the two \\ groups. However, when compared with Ser/ \\ Ser and Ser/Cys genotypes combined, we \\ found that the homozygous Cys/Cys genotype \\ was more prevalent in pterygium patients than \\ controls $(P=0.024)$ with the odds ratio being \\ 2.2 (95\% CI: 1.1-4.5). In the pterygium group, \\ the mean age of patients with the Cys/Cys \\ genotype was younger than those with the \\ other two genotypes $(P=\mathbf{0 . 0 2 5})$. \\ Conclusions Our findings suggest that the \\ $1245 \mathrm{C} \rightarrow \mathrm{G}$ transition in exon 7 of the hOGG1 \\ gene, which results in Ser326Cys substitution \\ of the enzyme, might play a role in the \\ susceptibility of humans to pterygium.
}

$\mathrm{H}-\mathrm{C} \mathrm{Kau}{ }^{1,2}, \mathrm{C}-\mathrm{C} \mathrm{Tsai}^{3,4}, \mathrm{~W}-\mathrm{M} \mathrm{Hsu}{ }^{3,4}, \mathrm{~J}-\mathrm{H} \mathrm{Liu}{ }^{5}$ and $\mathrm{Y}-\mathrm{H} \mathrm{Wei}^{1,6}$

IInstitute of Clinical Medicine National Yang-Ming University, Taiwan Republic of China

${ }^{2}$ Department of Ophthalmology Taoyuan Veterans Hospital, Taiwan Republic of China

Keywords: genotype; hOGG1; polymorphism; pterygium

\section{Introduction}

Pterygia are common ocular surface lesions, which are especially prevalent in the population of tropical and subtropical areas. The prevalence of pterygia in adults over 40 years old in Southeast Asia has been estimated to be $7 \% .^{1}$ Pterygia may invade adjacent cornea and cause astigmatism and visual impairment. Surgical excision is the current treatment strategy if the visual axis is threatened or in cases of extreme irritation. However, the recurrent rate is still significant.

Pterygium was regarded as an ocular degenerative disease with pathological evidence of degeneration in the conjunctival collagen fibre. However, pterygia display some tumourlike properties in their clinical appearance of local invasion and epithelial cell metaplasia. ${ }^{2}$ Furthermore, the presence of oncogenic viruses, such as the human papilloma virus and herpes simplex virus, ${ }^{3,4}$ loss of heterozygosity, ${ }^{5}$ and abnormal p53 expression in pterygium lesions, ${ }^{6,7}$ all suggest the possible neoplastic nature of pterygium. A model of pterygium formation is then proposed, in which genetic predisposition, environmental factors, and viral infections may participate in the multistep process. ${ }^{8}$ However, there is still a lack of strong evidence regarding the genetics of the disease.

Although the pathogenesis of pterygium is still uncertain, some risk factors including ultraviolet (UV) exposure, ${ }^{9-12}$ age,${ }^{1,11}$ race, ${ }^{13}$ and family history ${ }^{14}$ have been proposed. Among these risk factors, UV exposure has been documented to have strong correlation with
${ }^{3}$ Department of Ophthalmology National Yang-Ming University, Taiwan Republic of China

${ }^{4}$ Department of Ophthalmology, Taipei Veterans General Hospital, Taiwan Republic of China

${ }^{5}$ Department of Ophthalmology Cheng Hsin

Rehabilitation Medical

Center

Taiwan, Republic of

China

${ }^{6}$ Department of Biochemistry and Center for Cellular and Molecular Biology National, Yang-Ming University, Taipei, Taiwan Republic of China

Correspondence:

Y-H Wei

Department of Biochemistry and Center for Cellular and Molecular Biology National Yang-Ming University, Taiwan 155, Li-Nong Street Sec.2

Taipei, Taiwan 112 Republic of China Tel: +886228267118 Fax: + 886228264843

E-mail: joeman@ym.edu.tw

Received: 3 April 2003

Accepted: 21 July 2003

Published online:

9 January 2004 
pterygium, but the mechanism by which UV light induces pterygium remains elusive. Nolan et al ${ }^{15}$ demonstrated that heparin-binding epidermal growth factor-like growth factor, a potent mitogen, was localized in pterygium tissue, and was significantly induced by UVB in pterygium-derived epithelial cells. Lu et $a l^{16}$ has proposed photo-damage in pterygium from the point of view of lipid peroxidation. UV irradiation may damage various cellular biomolecules, especially the DNA. Once DNA is modified without proper repair, altered genetic expression and even mutation may occur. The photooxidative damage to DNA results in pyrimidine dimers or various DNA modifications such as 8-hydroxy2'-deoxyguanosine (8-OHdG), strand breaks, sites of base loss, and DNA-protein crosslinks. ${ }^{17}$ Among all the photo-oxidative DNA products, the $8-\mathrm{OHdG}$ is regarded a sensitive and stable biomarker for evaluating the degree of DNA damage. ${ }^{18}$ The $8-\mathrm{OHdG}$ is highly mutagenic, because it frequently mispairs with adenine during DNA replication, ultimately giving rise to the $\mathrm{G}-\mathrm{C}$ to $\mathrm{T}-\mathrm{A}$ transversion mutations. ${ }^{19}$ The increase in 8-OHdG content in DNA has been shown to increase cancer risks. ${ }^{20}$

In human genome, there is a base excision repair system to correct 8-OHdG. The human 8-oxoguanine glycosylase I (hOGG1) is the key component responsible for the removal of $8-\mathrm{OHdG}$ in DNA. ${ }^{21}$ Previous genetic studies have revealed the presence of several polymorphisms within the hOGG1 locus. ${ }^{22,23}$ Among them, a common polymorphism has been shown to have a functional difference. A transition of $C$ to $G$ at nucleotide position 1245 in exon 7 of the hOGG1 gene is associated with the substitution of cysteine for serine at codon 326. It has been proved in an Escherichia coli complementation assay ${ }^{23}$ that the DNA repair activity of hOGG1-Cys ${ }^{326}$ protein is lower than that of hOGG1Ser $^{326}$. The hOGG1 Cys/Cys homozygosity has been reported to increase the risks of lung cancer, ${ }^{24}$ oesophageal cancer, ${ }^{25}$ orolaryngeal cancer, ${ }^{26}$ and stomach cancer. ${ }^{27}$ Additionally, some studies revealed the impact of this genetic polymorphism on patients suffering from chronic oxidative stress. ${ }^{28,29}$ In this study, we tried to investigate whether the hOGG1 genetic polymorphism is associated with the susceptibility of humans to pterygium.

\section{Materials and methods}

We recruited pterygium patients from the Department of Ophthalmology at the Taipei Veterans General Hospital from August 2002 to February 2003. The diagnosis of pterygium was established according to the clinical finding of a wing-shaped fold of conjunctiva and fibrovascular tissue that has invaded the adjacent cornea for which there was no alternative explanation (for example, trauma). Patients with ocular diseases other than pterygium were excluded from this study. The volunteers in the control group were healthy subjects recruited when they attended their routine health examination. All the subjects were nonrelated. Clinical examination and diagnosis were all performed by the same ophthalmologist. To eliminate the factors that might affect the systemic oxidative stress, the exclusion criteria for the patients and control subjects were a history of smoking, taking vitamins or nutrition supplements, as well as those suffering from benign or malignant tumours, inflammatory or infectious diseases. Each eligible subject was interviewed to obtain data on age, sex, and occupational history. For analysis, the occupations were combined into two groups: outdoor (soldier, farmer, fisherman, and postman) or indoor (professional, office worker, teacher, and housekeeper). Our Institutional Review Board approved this study, and informed consent was obtained from all subjects after explanation of the nature and possible consequences of the study.

Each subject donated $5 \mathrm{ml}$ of blood that was collected in a heparinized tube. The genomic DNA was prepared by serial phenol/chloroform extraction and then ethanol precipitation. The genotype of hOGG1 was determined by the polymerase chain reaction-restriction fragmentlength polymorphism (PCR-RFLP) analysis. An aliquot of $100 \mathrm{ng}$ of genomic DNA was added to a $50 \mu \mathrm{l}$ PCR mixture containing $10 \mathrm{mM}$ Tris- $\mathrm{HCl}(\mathrm{pH} 8.0), 50 \mathrm{mM} \mathrm{KCl}$, $0.1 \%$ Triton $\mathrm{X}-100,2 \mathrm{mM} \mathrm{MgCl}, 200 \mu \mathrm{M}$ of each dNTP, 100 pmol of each primer, and $1 \mathrm{U}$ of Taq DNA polymerase (Biotools; B \& M Labs, Madrid, Spain). PCR was performed using the primers described by Kohno et $a{ }^{\prime},{ }^{11}$ that is, $5^{\prime}$-AGGGGAAGGTGCTTGGGGGAA-3' as the forward primer and 5'-ACTGTCACTA

GTCTCACCAG-3' as the reverse primer. The thermal profile consisted of 35 cycles of denaturation at $94^{\circ} \mathrm{C}$ for $15 \mathrm{~s}$, annealing at $58^{\circ} \mathrm{C}$ for $15 \mathrm{~s}$, and extension at $72^{\circ} \mathrm{C}$ for $40 \mathrm{~s}$, preceded by an initial denaturation step at $94^{\circ} \mathrm{C}$ for $2 \mathrm{~min}$ and followed by a terminal extension at $72{ }^{\circ} \mathrm{C}$ for $5 \mathrm{~min}$. Following PCR, $10 \mu \mathrm{l}$ of the product was subjected to Fnu4HI digestion (2.5 U of enzyme in a $15 \mu \mathrm{l}$ digest). The presence of $C$ to $G$ transversion at nucleotide position 1245 created an Fnu4HI recognition site, which led to digestion of the $200 \mathrm{bp}$ PCR product into two DNA fragments of $100 \mathrm{bp}$. Fnu $4 \mathrm{HI}$ digests of PCR products were separated by electrophoresis on a 3\% agarose gel, followed by staining with ethidium bromide.

The distributions of categorical variables (eg, gender, occupation type, and genotype) in patients and control groups were compared with $\chi^{2}$-test. The continuous variable (eg, age) was examined using the Student's $t$-test. A $P$-value less than 0.05 was considered to be 
statistically significant. The odds ratio (OR) and 95\% confidence interval (CI) were calculated and adjusted for age, gender, and occupation type using logistic regression.

\section{Results}

In total, 70 patients with pterygium and 86 healthy subjects were enrolled in this study. The mean age in the pterygium patients and control groups were $71.7 \pm 10.1$ and $68.3 \pm 13.2$ years, respectively. The proportions of the male gender in two groups were 85.7 and $74.4 \%$, respectively. The proportions of outdoor occupation were 77.1 and $64.0 \%$, respectively. The differences between pterygium and control groups for age, sex, and occupation type were not statistically significant.

The allelic frequencies for the C1245 (Ser) and G1245 (Cys) genotype of the hOGG1 gene were found to be 0.414 and 0.586 among the pterygium patient group, compared with 0.465 and 0.535 among the control group $(P=0.369)$ (Table 1$)$. The distributions of hOGG1 genotypes among pterygium patients (Ser/Ser, 22.9\%; Ser/Cys, 37.1\%; Cys/Cys, $40.0 \%$ ) were different from those of the control subjects (Ser/Ser, 16.3\%; Ser/Cys, $60.5 \%$; Cys/Cys, $23.3 \%)(P=0.014)$. The frequency of the Cys/Cys genotype in pterygium patients was higher than that of normal controls. When compared with Ser/ Ser and Ser/Cys genotypes combined, homozygous Cys/Cys genotype significantly increased the risk of developing pterygium $(P=0.024)$, with the OR adjusted for age, sex, and occupation type being 2.2 (95\% CI: 1.1-4.5) (Table 1).

Among the pterygium patients, the mean age of patients with Cys/Cys genotype $(67.8 \pm 14.1$ years old) was significantly lower than those with Ser/Ser and

Table 1 Allelic frequency, genotype frequency, and OR of hOGG1 Ser326Cys among pterygium patients and control groups

\begin{tabular}{|c|c|c|c|}
\hline & $\begin{array}{l}\text { Control group } \\
\quad(\mathrm{n}=86)\end{array}$ & $\begin{array}{l}\text { Pterygium group } \\
\quad(\mathrm{n}=70)\end{array}$ & P-value \\
\hline \multicolumn{4}{|l|}{ Allelic frequency } \\
\hline Cys & 0.535 & 0.586 & 0.369 \\
\hline Ser & 0.465 & 0.414 & \\
\hline \multicolumn{4}{|l|}{$\begin{array}{l}\text { Genotype } \\
\text { distribution }\end{array}$} \\
\hline Cys/Cys & $20(23.3 \%)$ & $28(40.0 \%)$ & 0.024 \\
\hline $\begin{array}{l}\text { Ser/Cys and } \\
\text { Ser/Ser }\end{array}$ & $66(76.7 \%)$ & $42(60.0 \%)$ & \\
\hline OR $(95 \% \mathrm{CI})^{\mathrm{a}}$ & & $2.2(1.1-4.5)$ & 0.369 \\
\hline
\end{tabular}

Ser/Cys genotypes combined $(74.3 \pm 4.8$ years old $)$ $(P=0.025)$

\section{Discussion}

Our findings showed that the hOGG1 Ser326Cys polymorphism was associated with the susceptibility of humans to pterygium. Individuals who carried the homozygous Cys/Cys genotype were at a 2.2-fold higher risk to develop pterygium. Besides, the mean age of pterygium patients with Cys/Cys genotype was younger than those with the other two genotypes combined.

Since the genetic polymorphism of hOGG1 has been associated with many kinds of neoplasia, it is reasonable to expect it to play a role in human cancer susceptibility. ${ }^{24-27}$ In order not to interfere with our analysis of hOGG1 polymorphism on the susceptibility to the pterygium, the subjects suffering from benign or malignant tumours were excluded. On the other hand, owing to the fact that the veterans accounted for approximately $50 \%$ of our patients, there were higher proportions of male sex and outdoor occupation type in our patients and controls. However, the association between hOGG1 Ser326Cys polymorphism and pterygium was still statistically significant after OR was adjusted for age, sex, and occupation type.

The hOGG1 is a specific DNA glycosylase/apurinic lyase that is responsible for the removal of $8-\mathrm{OHdG}$ in DNA. The hOGG1 molecule can recognize 8-OHdG, and then catalyse both the release of $8-\mathrm{OHdG}$ and the cleavage of DNA at the resulting apurinic site. ${ }^{21}$ The enzyme itself is very sensitive to UVB irradiation, which causes photolysis of tryptophan residues at the active site and leads to hOGG1 inactivation. ${ }^{30}$ It has been demonstrated that a CG polymorphism at nucleotide position 1245 in exon 7 of the hOGG1 gene results in an amino-acid substitution from serine to cysteine at residue 326. According to the functional study by Kohno et al, ${ }^{23}$ the Cys326 allele is about seven-fold less capable of complementing a repair-deficient $E$. coli strain than the Ser326 allele in an in vitro complementation assay. Besides, the inactivation of hOGG1 increases the frequency of $\mathrm{G}-\mathrm{C}$ to $\mathrm{T}-\mathrm{A}$ transversion mutations in Saccharomyces cerevisiae. ${ }^{31}$ It could be possible that the Cys326 allele has reduced enzymatic activity toward 8-OHdG or increased sensitivity to UVB inactivation. As a result, individuals bearing the homozygous Cys/Cys genotype might allow the persistent presence of $8-\mathrm{OHdG}$ in genomic DNA and accelerate the accumulation of $\mathrm{G}-\mathrm{C}$ to T-A mutations, which may lead to an increased susceptibility of an individual to develop pterygium. Further studies on the effect of the genetic polymorphism on the enzyme function of hOGG1 in vivo are warranted. 
In conclusion, our study demonstrated for the first time that Ser326Cys polymorphism in hOGG1 is associated with the risk of pterygium in Chinese. In view of the complexity in the aetiology of pterygium, no single genetic marker is sufficient for prediction of the risk of this disease. Therefore, a panel of susceptibility biomarkers including genetic polymorphisms in other DNA repair pathways or photo-oxidative reactions is warranted to define individuals at a high risk of developing pterygium.

\section{Acknowledgements}

Supported by a grant (NSC 91-2320-B010-069) from the National Science Council, Taiwan. None of the authors have any commercial interest in the materials mentioned herein.

\section{References}

1 Wong TY, Foster PJ, Johnson GJ, Seah SK, Tan DT. The prevalence and risk factors for pterygium in an adult Chinese population in Singapore: the Tanjong Pagar survey. Am J Ophthalmol 2001; 131: 176-183.

2 Chan CM, Liu YP, Tan DT. Ocular surface changes in pterygium. Cornea 2002; 21: 38-42.

3 Gallagher MJ, Giannoudis A, Herrington CS, Hiscott P. Human papillomavirus in pterygium. Br J Ophthalmol 2001; 85: 782-784.

4 Detorakis ET, Sourvinos G, Spandidos DA. Detection of herpes simplex virus and human papilloma virus in ophthalmic pterygium. Cornea 2001; 20: 164-167.

5 Detorakis ET, Sourvinos G, Tsamparlakis J, Spandidos DA. Evaluation of loss of heterozygosity and microsatellite instability in human pterygium: clinical correlations. $\mathrm{Br}$ J Ophthalmol 1998; 82: 1324-1328.

6 Tan DT, Lim AS, Goh HS, Smith DR. Abnormal expression of the p53 tumor suppressor gene in the conjunctiva of patients with pterygium. Am J Ophthalmol 1997; 123: 404-405.

7 Ueda Y, Kanazawa S, Kitaoka T, Dake Y, Ohira A, Ouertani $\mathrm{AM}$ et al. Immunohistochemical study of p53, p21 and PCNA in pterygium. Acta Histochem 2001; 103: 159-165.

8 Detorakis ET, Drakonaki EE, Spandidos DA. Molecular genetic alterations and viral presence in ophthalmic pterygium. Int J Mol Med 2000; 6: 35-41.

9 Threlfall TJ, English DR. Sun exposure and pterygium of the eye: a dose-response curve. Am J Ophthalmol 1999; 128: 280-287.

10 Tang FC, Chen SC, Lee HS, Lin WF, Chou MC, Lee MC. Relationship between pterygium/pinguecula and sunlight exposure among postmen in central Taiwan. Chin Med J 1999; 62: 496-502.

11 Saw SM, Banerjee K, Tan D. Risk factors for the development of pterygium in Singapore: a hospital-based case-control study. Acta Ophthalmol Scand 2000; 78: 216-220.

12 Gazzard G, Saw SM, Farook M, Koh D, Widjaja D, Chia SE et al. Pterygium in Indonesia: prevalence, severity and risk factors. Br J Ophthalmol 2002; 86: 1341-1346.
13 Luthra R, Nemesure BB, Wu SY, Xie SH, Leske MC. The Barbados Eye Studies Group. Frequency and risk factors for pterygium in the Barbados Eye Study. Arch Ophthalmol 2001; 119: $1827-1832$.

14 Booth F. Heredity in one hundred patients admitted for excision of pterygia. Aust N Z J Ophthalmol 1985; 13: 59-61.

15 Nolan TM, DiGirolamo N, Sachdev NH, Hampartzoumian $\mathrm{T}$, Coroneo MT, Wakefield D. The role of ultraviolet irradiation and heparin-binding epidermal growth factorlike growth factor in the pathogenesis of pterygium. Am J Pathol 2003; 162: 567-574.

16 Lu L, Wang R, Song X. Pterygium and lipid peroxidation. Chin J Ophthalmol 1996; 32: 227-229.

17 Kielbassa C, Roza L, Epe B. Wavelength dependence of oxidative DNA damage induced by UV and visible light. Carcinogenesis 1997; 18: 811-816.

18 Kasai H. Analysis of a form of oxidative DNA damage, 8-hydroxy-2' - deoxyguanosine, as a marker of cellular oxidative stress during carcinogenesis. Mutat Res 1997; 387: 147-163.

19 Cheng KC, Cahill DS, Kasai H, Nishimura S, Loeb LA. 8-Hydroxyguanine, an abundant form of oxidative DNA damage, causes GT and AC substitutions. J Biol Chem 1992; 267: 166-172.

20 Greim H, Csanady G, Filser JG, Kreuzer P, Schwarz L, Wolff $\mathrm{T}$ et al. Biomarkers as tool in human health risk assessment. Clin Chem 1995; 41: 1804-1808.

21 Bruner SD, Norman DP, Verdine GL. Structural basis for recognition and repair of the endogenous mutagen 8-oxoguanine in DNA. Nature 2000; 403: 859-866.

22 Ishida T, Takashima R, Fukayama M, Hamada C, Hippo Y, Fujii T et al. New DNA polymorphisms of human MMH/ OGG1 gene: prevalence of one polymorphism among lungadenocarcinoma patients in Japanese. Int J Cancer 1999; 80: 18-21.

23 Kohno T, Shinmura K, Tosaka M, Tani M, Kim SR, Sugimura $\mathrm{H}$ et al. Genetic polymorphisms and alternative splicing of the hOGG1 gene, that is involved in the repair of 8hydroxyguanine in damaged DNA. Oncogene 1998; 16: 3219-3225.

24 Le Marchand L, Donlon T, Lum-Jones A, Seifried A, Wilkens LR. Association of the hOGG1 Ser326Cys polymorphism with lung cancer risk. Cancer Epidemiol Biomarkers Prevent 2002; 11: 409-412.

25 Xing DY, Tan W, Song N, Lin DX. Ser326Cys polymorphism in hOGG1 gene and risk of esophageal cancer in a Chinese population. Int J Cancer 2001; 95: 140-143.

26 Elahi A, Zheng Z, Park J, Eyring K, McCaffrey T, Lazarus P. The human OGG1 DNA repair enzyme and its association with orolaryngeal cancer risk. Carcinogenesis 2002; 23: 1229-1234.

27 Takezaki T, Gao CM, Wu JZ, Li ZY, Wang JD, Ding JH et al. hOGG1 Ser(326)Cys polymorphism and modification by environmental factors of stomach cancer risk in Chinese. Int J Cancer 2002; 99: 624-627.

28 Tarng DC, Tsai TJ, Chen WT, Liu TY, Wei YH. Effect of human OGG1 1245CG gene polymorphism on 8-hydroxy2'-deoxyguanosine levels of leukocyte DNA among patients undergoing chronic hemodialysis. J Am Soc Nephrol 2001; 12: 2338-2347.

29 Zhai R, Liu G, Ge X, Yang C, Huang C Wu C et al. Genetic polymorphisms of MnSOD, GSTM1, GSTT1, and OGG1 in coal workers' pneumoconiosis. J Occup Environ Med 2002; 44: 372-377. 
30 van der Kemp PA, Blais JC, Bazin M, Boiteux S, Santus R. Ultraviolet-B-induced inactivation of human OGG1, the repair enzyme for removal of 8-oxoguanine in DNA. Photochem Photobiol 2002; 76: 640-648.
31 Thomas D, Scott AD, Barbey R, Padula M, Boiteux S. Inactivation of OGG1 increases the incidence of G:CT:A transversions in Saccharomyces cerevisiae: evidence for endogenous oxidative damage in DNA in eukaryotic cells. Mol Gen Genet 1997; 254: 171-178. 